\title{
DEVELOPMENT AND EFFICACY A TRIVALENT VACCINE AGAINST BACTERIAL PATHOGENS in RAINBOW TROUT, Oncorhynchus mykiss
}

\author{
Aysegul Kubilay ${ }^{1}$, Pinar Yildirim ${ }^{1}$ \\ ${ }^{I}$ Department of Aquaculture, Faculty of Fisheries, SuleymanDemirel University, \\ Isparta, TURKEY, 32260
}

\begin{abstract}
In this study, a new trivalent vaccine was developed and evaluated the efficacy with adjuvants (Freund's Complete Adjuvant and glucan) and non-adjuvanted vaccine in rainbow trout(20g) against to pathogens; L. garvieae, V. anguillarumand $Y$. ruckeri. For this purpose, four experimental groups were formed including control. The vaccine was prepared formalin-killed bacteria and then combined with adjuvants that were given into fish by intraperitoneal injection. Control fish were received only phosphate buffered saline-PBS. A booster was applied the fish which were vaccinated with a nonadjuvanted vaccine 21 days post-injection. All the groups were challenged against three pathogens at day $30^{\text {th }}, 90^{\text {th }}, 120^{\text {th }}$ and $270^{\text {th }}$ post vaccination. The results showed that fish had a high level of protection of pathogens in the vaccinated groups up to 9 months. The level of protection was calculated by obtaining the Relative Percent Survival (RPS). RPS was determinedas in the non adjuvanted plus a booster injected group: Y.ruckeri 96,77\%, V.anguillarum 100\%; L.garvieae 96,66\%, Glucan: Y.ruckeri 93,54\%; V.anguillarum 96,77\%, L.garvieae 93,33 \%, FCA :Y.ruckeri 100\%, V.anguillarum $87,09 \%$, L.garvieae $93,33 \%$ at $270^{\text {th }}$ day. The efficacy of the vaccine has been found to provide long-term protection against to three pathogens in the experimental condition.
\end{abstract}

Key Words: Rainbow trout, Yersinia ruckeri, Lactococcus garvieae, Listonella(Vibrio) anguillarium, vaccine, adjuvant, Long-term protection 